\title{
Cord blood delta neutrophil index values of term neonates
}

\author{
Melek Büyükeren ${ }^{1}$, Hasan Tolga Çelik¹, Oytun Portakal², Erdem Fadıloğluఠ, \\ Gökçen Örgüll ${ }^{3}$, Şule Yiğit ${ }^{1}$, M. Sinan Beksaç ${ }^{3}$, Murat Yurdakök ${ }^{1}$ \\ ${ }^{1}$ Division of Neonatology, Department of Pediatrics, ${ }^{2}$ Department of Biochemistry, ${ }^{3}$ Division of Perinatology, Department of Obstetrics \\ and Gynecology, Hacettepe University Faculty of Medicine, Ankara, Turkey.
}

\begin{abstract}
In this study, we aimed to demonstrate cord blood immature granulocyte (IG) count and delta neutrophil index (DNI) values for term neonates. This retrospective study consisted of 126 term newborns born between July 2017 and December 2017. Cord blood samples were collected during delivery and IG count together with DNI values were obtained. "Beckman Coulter DXH800 System Hematology Analyzer" was used for analysis and calculations. The median DNI value was found to be 1.0 (interquantile range(IQR) $0.5-1.8 \%$ ) and the median gestational age at delivery was 38.4 (IQR 37.6-39.0) weeks. The median birth weight and IG count were 3250 (IQR 2955-3593) $\mathrm{g}$ and 66 (IQR 26.5-112.3)/ $\mathrm{mm}^{3}$, respectively. In conclusion, we believe that determining the normal laboratory reference values of IG count or DNI, which are important potential diagnostic markers for neonatal sepsis, will contribute to future studies on the diagnosis of neonatal sepsis.
\end{abstract}

Key words: cord blood, neonate, immature granulocyte ratio, delta neutrophil index, neonatal sepsis.

Neonatal sepsis is one of the major causes of mortality and severe morbidity in neonatal intensive care units. The overall incidence of neonatal sepsis ranges from one to five cases per 1000 live births. ${ }^{1}$ Due to its rapid and fatal course, early diagnosis and immediate initiation of appropriate antimicrobial therapy are critical. Globally, neonatal sepsis and other severe infections accounted for approximately 15 percent of all neonatal deaths. ${ }^{1}$

The clinical signs and symptoms of sepsis in newborns are nonspecific. The gold standard diagnostic method for sepsis is the isolation of the causative microorganism (bacterium or fungus) in one or more blood cultures. ${ }^{2}$ However, because the culture results are not immediately availiable and the procedure has certain limitations in newborns, blood cultures are not that useful for early diagnosis of sepsis during the neonatal period. ${ }^{3}$ This

$凶 \quad$ Erdem Fadıloğlu

erdemfadiloglu@hacettepe.edu.tr

Received 15th March 2019; revised 15th April 2019; accepted 22nd April 2019 situation has led to the development of several laboratory methods to facilitate the rapid and accurate diagnosis of neonatal sepsis. The most commonly used biomarkers are the immatureto-total neutrophil ratio (I/T), procalcitonin, C-reactive protein and interleukin-6 values.-6 Moreover, immature granulocyte (IG) ratio and delta neutrophil index (DNI) values seem to be potential predictive markers for neonatal sepsis. ${ }^{7,8}$ DNI is defined as immature granulocyte fraction provided by a blood cell analyzer which is determined by subtracting the fraction of mature polymorphonuclear leukocytes from the sum of myeloperoxidasereactive cells. ${ }^{9}$ IG count and DNI are reported to be used widely in diagnosis of sepsis and determination of disease severity in critically ill patients. $^{10}$

Recent technological improvements have enabled automated hematology analyzers to determine the IG count and fraction. The term IG is used to describe the myelocytes, promyelocytes and metamyelocytes (i.e., neutrophil precursors) that are found in the bone marrow but not in the peripheral blood 
after the neonatal period. ${ }^{11}$ On the other hand, it has been reported that immature neutrophil forms enter the circulation during infection..$^{10} \mathrm{In}$ addition to providing results in a shorter period of time, automatic IG counts are more accurate and precise than manual counts. ${ }^{11}$ Cord blood (CB) IG count and DNI are candidates to be used as sepsis markers. However, the normal range of these counts in neonates has not yet been established for $\mathrm{CB}$.

We believe that determining the normal laboratory reference values of IG count and DNI values as important potential diagnostic markers for neonatal sepsis will contribute to future studies on the diagnosis of neonatal sepsis.

\section{Material and Methods}

This retrospective study consisted of 126 term normal pregnancies that were delivered between July 2017 and December 2017. The inclusion criteria for $\mathrm{CB}$ sampling in this study were as follows: 1 ) Presence of written maternal informed consent for $\mathrm{CB}$ collection; 2) Absence of familial inherited diseases; 3) Negative hepatitis B, hepatitis C, Human immunodeficiency virus (HIV) and human T-cell leukemia virus type-I/II (HTLV) serology; 4) Term pregnancy; 5) No history of early rupture of membrane; 6) Absence of clinical and/or laboratory findings suggesting infection; 7) Absence of prenatally detected chromosomal anomalies and congenital abnormalities in the fetus; 8) Absence of placental abnormalities; 9) Absence of multiple gestations; 10) Absence of maternal chronic inflammatory diseases; and 11) Absence of maternal metabolic and immunological disorders. Pregnant women who did not meet these criteria were excluded from CB sampling.

Cord blood samples were collected from the umblical vein into EDTA tubes after delivery of the fetus and IG count together with DNI values were obtained. The complete blood count data was obtained using a UniCel DxH
800 hematology analyzer (Beckman Coulter, Inc., Brea, CA, USA), and the DNI values were automatically calculated by this device. The UniCel DxH 800 uses electrical impedance, radiofrequency conductivity, and volume, conductivity and multiangle light scattering to count and distinguish between the leukocyte subpopulations. ${ }^{12}$

A total of 179 healthy full-term neonates (born between $37^{0 / 7}$ weeks and $42^{6 / 7}$ weeks of gestation) were initially recruited for this research. Of those, fifty-three neonates suspected of having sepsis and/or with missing laboratory data were excluded. Thus, the remaining 126 subjects were included in this study.

Necessary clinical and demographic data were drawn from the electronic registry of Hacettepe University Hospital. All statistical analyses were performed using SPSS version 23.0. A descriptive analysis was performed for the whole cohort and non-parametric tests were used according to performed visual and statistical normality tests. Median values were given for whole parameters and Interquartile Ranges were given for the distrubition of the cases. Prior to conducting this study, ethical approval was obtained from the Hacettepe University Ethics Committee (GO-17/824-29).

\section{Results}

The demographic and neonatal characteristics of the 126 patients included in this study are presented in Table I. The median gestational

Table I. Demographic and neonatal data.

\begin{tabular}{ll}
\hline Characteristics & $\mathrm{n}(\%)$ \\
\hline Gender (M/F) & $57 / 69(45.2 \% / 54.8 \%)$ \\
Gestational age (weeks) $^{*}$ & $38.4 \pm 1.0(38.4 ; 37.6-39.0)$ \\
Apgar score (5 min)* & $9.7 \pm 0.8(10 ; 10-10)$ \\
Resuscitation at birth & $4(3.17 \%)$ \\
Birthweight (g) & $3272 \pm 452(3250 ; 2955-3593)$ \\
SGA/LGA & $5 / 4(3.96 \% / 3.17 \%)$ \\
\hline
\end{tabular}

*Mean \pm standard deviation (median; $25^{\text {th }}-75^{\text {th }}$ percentile) SGA: small for gestational age, LGA: large for gestational age 
Table II. Complete blood count values.

\begin{tabular}{ll}
\hline Parameters & Mean \pm SD $\left(\right.$ median; $25^{\text {th }}-75^{\text {th }}$ percentile $)$ \\
\hline Hemoglobin $(\mathrm{g} / \mathrm{dL})$ & $14.8 \pm 1.6(14.9 ; 13.5-15.8)$ \\
Hematocrit $(\%)$ & $46.2 \pm 5.3(46.0 ; 42.0-49.6)$ \\
Platelet count $(/ \mu \mathrm{L})$ & $239730 \pm 70077(244500 ; 203500-278500)$ \\
White blood cell count $(/ \mu \mathrm{L})$ & $11393 \pm 4120(10600 ; 8900-13250)$ \\
ANC $(/ \mu \mathrm{L})$ & $6550 \pm 3240(5959.5 ; 4483.8-7763.0)$ \\
DNI $(\%)$ & $1.3 \pm 1.0(1.0 ; 0.5-1.8)$ \\
IGC $(/ \mu \mathrm{L})$ & $94 \pm 127(66 ; 26.5-112.3)$ \\
\hline
\end{tabular}

ANC: absolute neutrophil count, DNI: delta neutrophil index, IGC: immature granulocyte count.

age was 38.4 weeks (interquartile range (IQR) 37.6-39.0 weeks), and the median birth weight was 3250 (IQR 2955-3593) g. Fifty-seven (45\%) of the neonates were males (Table I). Complete blood count values of the neonates are shown in Table II. The median DNI value was found to be 1.0 (IQR 0.5-1.8\%). Immature granulocyte count was 66 (IQR 26.5-112.3)/ $/ \mathrm{mm}^{3}$.

\section{Discussion}

Sepsis is common in neonatal intensive care units, and it is responsible for significant mortality and morbidity. When treating sepsis, it is critical that therapy targeting the causative agent is initiated as early as possible. However, isolating the causative agent requires time, and it is not always feasible in newborns. Therefore, sepsis markers are more important in this patient group. ${ }^{2,3}$ One of the most significant markers is the left shift of neutrophils in the peripheral blood smear, which refers to an increase in the $\mathrm{I} / \mathrm{T}$ ratio. A value greater than 0.2 reflects an increase in the number of immature neutrophils, which is suggestive of sepsis. ${ }^{13}$ Although the $\mathrm{I} / \mathrm{T}$ ratio is a valuable marker in the diagnosis of neonatal sepsis, it can be unreliable since it is determined based on counting 100 cells in a peripheral blood smear. Therefore, its accuracy is directly proportional to the observer's (counter's) experience. ${ }^{12-14} \mathrm{We}$ believe that novel neonatal sepsis markers are necessary for better clinical practice.

Nahm et al. ${ }^{15}$ reported no statistical difference between the immature neutrophil counts obtained from an automated hematology analyzer and those obtained from a hematologist who manually counted 200 cells from a peripheral blood smear. Lee et al. ${ }^{7}$ observed a statistically significant difference in the DNI values between 24 newborns with sepsis and a control group consisting of 48 newborns without sepsis (mean gestational age: $37.0 \pm 7.2$ weeks, mean birth weight: $2910 \pm 650 \mathrm{~g}$ ), with the sepsis group showing higher values at the time of diagnosis, 24 hours after the diagnosis, and 72 hours after the diagnosis. In the same study, the DNI values at the time of diagnosis in the septic newborns who died, those who survived and the control group were $6.5 \pm 2.4 \%, 3.7 \pm 1.8 \%$ and $1.1 \pm 0.7 \%$, respectively. Cimenti et al. ${ }^{12}$ determined a DNI cut-off value of $1.3 \%$ based on the DNI values of 21 infants diagnosed with sepsis and 112 controls. In a study by Wiland et al. ${ }^{8}$, the IG percentage of infants with a mean gestational age of $39 \pm 1.6$ weeks and a mean birth weight of $3187 \pm 520 \mathrm{~g}$ ranged between $0 \%$ and $8.4 \%$ within the first 48 hours of life, and it was greater than $1 \%$ in $70 \%$ of the samples before 12 hours. Therefore, they concluded that the IG percentage threshold of $1 \%$ that is used to diagnose sepsis in adults and children is not suitable for newborns during the first 48 hours of life. In their study, Wang et al. ${ }^{16}$ reported that the I/T value was the highest at birth ( 0 hours) in newborns with very low birth weights.

In our study, the median DNI of the CB samples of 126 healthy full-term neonates with a median gestational age of 38.4 weeks (IQR 37.6-39.0 weeks) and a median birth weight of 3250 (IQR 
2955-3593)g was 1.0 (IQR 0.5-1.8\%). The lowest DNI value was $0 \%$ and the highest was $3.9 \%$. The median immature granulocyte count was 66(IQR 26.5-112.3) $/ \mathrm{mm}^{3}$ and it ranged from a minimum of $0 / \mathrm{mm}^{3}$ to a maximum of 1153 / $\mathrm{mm}^{3}$. The results of this preliminary study may be used in clinical practice for the early prediction of neonatal sepsis. We believe that, further studies are necessary in this field.

The limitations of this study were the lack of a neonatal sepsis group and the small number of cases. On the other hand, this was the first study to demonstrate the normal IG counts and fractions exclusively in the $\mathrm{CB}$ of healthy fullterm newborns. The fact that preterm infants were not taken into the study is one of the limitations.

We believe that determining the normal laboratory reference values of IG count and DNI values in $\mathrm{CB}$ as important potential diagnostic markers for neonatal sepsis will contribute to future studies on the diagnosis of neonatal sepsis.

\section{REFERENCES}

1. Oza S, Lawn JE, Hogan DR, Mathers C, Cousens SN. Neonatal cause-of-death estimates for the early and late neonatal periods for 194 countries: 2000-2013. Bull World Health Organ 2015; 93: 19-28.

2. Mukhopadhyay S, Puopolo KM. Risk assessment in neonatal early-onset sepsis. Semin Perinatol 2012; 36: 408-415.

3. Connell TG, Rele M, Cowley D, Buttery JP, Curtis N. How reliable is a negative blood culture result? Volume of blood submitted for culture in routine practice in a children's hospital. Pediatrics 2007; 119: 891-896.

4. Chauhan N, Tiwari S, Jain U. Potential biomarkers for effective screening of neonatal sepsis infections: an overview. Microb Pathog 2017; 107: 234-242.

5. Hedegaard SS, Wisborg K, Hvas AM. Diagnostic utility of biomarkers for neonatal sepsis--a systematic review. Infec Dis (Lond) 2015; 47: 117-124.
6. Tang BM, Eslick GD, Craig JC, McLean AS. Accuracy of procalcitonin for sepsis diagnosis in critically ill patients: systematic review and meta-analysis. Lancet Infect Dis 2007; 7: 210-217.

7. Lee SM, Eun HS, Namgung R, Park MS, Park KI, Lee C. Usefulness of the delta neutrophil index for assessing neonatal sepsis. Acta Paediatr (Oslo) 2013; 102: e13-e16.

8. Wiland EL, Sandhaus LM, Georgievskaya Z, Hoyen CM, O'Riordan MA, Nock ML. Adult and child automated immature granulocyte norms are inappropriate for evaluating early-onset sepsis in newborns. Acta Paediatr (Oslo, Norway: 1992) 2014; 103: 494-497.

9. Seok Y, Choi JR, Kim J, et al. Delta neutrophil index: a promising diagnostic and prognostic marker for sepsis. Shock (Augusta) 2012; 37: 242-246.

10. Park BH, Kang YA, Park MS, et al. Delta neutrophil index as an early marker of disease severity in critically ill patients with sepsis. BMC Infect Dis 2011; 11: 299.

11. Nierhaus A, Klatte S, Linssen J, et al. Revisiting the white blood cell count: immature granulocytes count as a diagnostic marker to discriminate between SIRS and sepsis--a prospective, observational study. BMC Immunol 2013; 14: 8.

12. Cimenti C, Erwa W, Müller W, Resch B. The role of immature granulocyte count and immature myeloid information in the diagnosis of neonatal sepsis. Neonatal Bacterial Infection: IntechOpen, 2013. http://dx.doi.org/10.5772/54531.

13. Wynn JL, Wong HR, Shanley TP, Bizzarro MJ, Saiman L, Polin RA. Time for a neonatal-specific consensus definition for sepsis. Pediatr Crit Care Med 2014; 15: 523-528.

14. Mahale R, Dutta S, Ahluwalia J, Kishore SS, Narang A. Baseline illness severity does not alter accuracy of neonatal sepsis screen. Am J Perinatol 2010; 27: 327-332.

15. Nahm CH, Choi JW, Lee J. Delta neutrophil index in automated immature granulocyte counts for assessing disease severity of patients with sepsis. Ann Clin Lab Sci 2008; 38: 241-246.

16. Wang J, Yu J, Fan J, He Y, Dong W, Wang Z. Evaluation of altitude-appropriate reference ranges for neutrophils in diagnosis of sepsis in very low birth weight infants: a multicenter retrospective study. PLoS One 2017; 12: e0171571. 